media but a lesser response in the BMFs correspondence columns. We are also interested to know why the study did not relate staging to outcome-for instance, in 74 women who had died by the time the study was under way. Was much less information on staging available for these women? registry and pathology records, we wonder whether the authors determined how often notes kept about treatment corresponded with the records of treatment or surgery recorded by other hospital departments. We have found considerable discrepancies, for example, between incidences of breast cancer recorded by registries and hospitals. Would this introduce bias?

Despite our queries about the research, we share Chouillet and colleagues' belief in the value of audit of treatment of breast cancer as a means of improving services for patients with the disease.

\section{ELIZABETH ROBERTS}

Director

LUCY BRANSTON Research officer

Breast Test Wales,

Cardiff CF1 9LJ

1 Chouillet AM, Bell CMJ, Hiscox JG. Management of breast cancer in southeast England. $B M f$ 1994;308:168-71. (15 January.)

\section{Hyperlipidaemia and hypothyroidism}

\section{Screen patients for hypothyroidism before treatment}

EDrroR,-M A Al-Jubouri and colleagues report a case of subclinical myopathy in association with hypothyroidism and simvastatin treatment. ${ }^{1}$ In line with existing guidelines, ${ }^{2}$ the authors highlighted the importance of excluding secondary causes of hypercholesterolaemia before starting hypolipidaemic treatment. Hypothyroidism is a well recognised cause of secondary hyperlipidaemia, and in our lipid clinic every new patient's thyroid stimulating hormone and thyroxine concentrations are screened irrespective of the presenting lipid pattern. We recently audited this practice and present our findings on the incidence of previously unrecognised hypothyroidism at presentation and the effect of thyroxine treatment on patients with hypothyroidism.

Of 1190 new referrals (623 men, 567 women), 11 were found to have biochemical hypothyroidism (thyroid stimulating hormone concentration $>6 \mathrm{mU} / \mathrm{l}$; thyroxine concentration $<50 \mathrm{nmol} / \mathrm{l}$ ) and 13 to have compensated biochemical hypothyroidism (thyroid stimulating hormone $>6 \mathrm{mU} / \mathrm{l}$; thyroxine $50-150 \mathrm{nmol} / \mathrm{)}$, giving incidences of $0.92 \%$ and $1.1 \%$ respectively. The thyroid stimulating hormone concentration was significantly higher and thyroxine concentration lower in biochemical hypothyroidism than com-
Having worked extensively with both cancer

pensated biochemical hypothyroidism, but other characteristics, including the lipid profile at presentation, were similar in both groups (table). While most patients had a type IIa lipid pattern, one patient with compensated biochemical hypothyroidism had a type III lipid pattern with apo E phenotype E2/2 and another had combined hyperlipidaemia with a triglyceride concentration of 12.5 $\mathrm{mmol} / \mathrm{m}$

All patients with biochemical hypothyroidism were treated with thyroxine $(100-150 \mu \mathrm{g} /$ day $)$ and rendered biochemically euthyroid, with normalisation of the lipid profile in eight. Five patients with compensated biochemical hypothyroidism received treatment with thyroxine because of further increases in their thyroid stimulating hormone concentration with or without a further fall in thyroxine concentration. Four of these required additional hypolipidaemic agents to achieve desirable lipid concentrations. None of the patients in either group had a raised creatine kinase value at presentation or during treatment.

Many hypothyroid patients may present with hypercholesterolaemia in the absence of clinical features, as shown by our population. Furthermore, our data suggest that the presenting lipid profile of demographic features cannot be used to distinguish between biochemical hypothyroidism and compensated biochemical hypothyroidism. The incidence of biochemical hypothyroidism in this series is lower than that reported in other populations in lipid clinics. ${ }^{34}$ The differences probably reflect local variations in the overall incidence of hypothyroidism or in the practices of the primary referral clinicians. Although the effect of thyroxine treatment on the lipid profile in biochemical hypothyroidism differs in various studies, ${ }^{5}$ our results indicate that thyroxine treatment alone should normalise lipid concentrations in most patients with biochemical hypothyroidism but that most patients with compensated biochemical hypothyroidism need additional hypolipidaemic agents. VEF CROWLEY Registrar A LAWAL Honorary registra C WEINKOVE Consultant chemical pathologist Department of Clinical Biochemistry,

Hope Hospital, Salford M6 8HD

1 Al-Jubouri MA, Briston PG, Sinclair D, Chinn RH, Young RM Myxoedema revealed by simvastatin induced myopathy. $B M \mathcal{F}$ 1994;308:588. (26 February.)

2 Betteridge DJ, Dodson PM, Durrington PN, Hughes EA, Laker MF, Nicholls DP, et al. Management of hyperlipidaemia guidelines of the British Hyperlipidaemia Association. Postgrad Med f 1993;69:359-69.

3 O'Kane MJ, Neeley RDG, Trimble ERT, Nicolls DP. The incidence of asymptomatic hypothyroidism in new referrals to a hospital lipid clinic. Ann Clin Biochem 1991;28:509-11.

4 Bruckert E, De Gennes JL, Dairou F, Turpin G. Frequency of hypothyroidism in a population of hyperlipidaemic subjects. Presse Med 1993;22:57-60.

5 Fowler PBS, Stubbs P. Asymptomatic hypothyroidism and hypercholesterolaemia. $₹ R$ Soc Med 1992;85:244-5.

Characteristics of hyperlipidaemic patients with biochemical hypothyroidism among 1190 patients referred to lipid clinic Values are means (ranges) except for thyroid stimulating hormone, which is expressed as median (range)

Biochemical hypothyroidism Compensated biochemical hypothyroidism

\begin{tabular}{lcc}
\hline No (M/F) & $4 / 7$ & $2 / 11$ \\
Mean age (years) & $57 \cdot 0(39-67)$ & $56 \cdot 9(37-72)$ \\
Body mass index & $26 \cdot 1(20-31)^{\star}$ & $25 \cdot 3(20-30)$ \\
Thyroid stimulating hormone (mU/l) & $44 \cdot 0(8 \cdot 1-50)^{\star}$ & $9 \cdot 8(6 \cdot 1-50)$ \\
Thyroxine (nmol/) & $39 \cdot 5(20-49)^{\star}$ & $77 \cdot 1(60-100)$ \\
Cholesterol (mmol/) & $8 \cdot 9(7 \cdot 9-10 \cdot 3)$ & $8 \cdot 81(6 \cdot 1-11 \cdot 2)$ \\
Triglycerides (mmol/) & $2 \cdot 1(1 \cdot 1-3 \cdot 3)$ & $3 \cdot 9(1 \cdot 3-12 \cdot 5)$ \\
High density lipoprotein cholesterol (mmol/l) & $1 \cdot 22(0 \cdot 83-1 \cdot 75)$ & $1 \cdot 37(0 \cdot 70-2 \cdot 1)$ \\
Low density lipoprotein cholesterol (mmol/) & $6 \cdot 4(4 \cdot 3-7 \cdot 7)$ & $5 \cdot 9(3 \cdot 7-6 \cdot 8)$ \\
No treated with thyroxine & 8 & 1 \\
No treated with thyroxine and hypolipidaemic agent & 3 & 4
\end{tabular}

$\star \mathrm{P}<0.0001$ compared with patients with compensated biochemical hypothyroidism.
Screening everyone would be expensive and unnecessary

EDrroR,-M A Al-Jubouri and colleagues' lesson of the week highlights the pitfalls of treating patients with hypercholesterolaemia without due consideration of secondary causes.'The lesson offered by the authors-that myxoedema should be considered in people with hypercholesterolaemia before hypolipidaemic treatment is started-may not, however, provide the answer. To perform thyroid tests for all patients who have hypercholesterolaemia would be unnecessary and not cost effective. Previous work suggests that biochemical assessment of thyroid status in patients with a total cholesterol concentration $\geqslant 8 \mathrm{mmol} / /$ would be beneficial. ${ }^{2}$ If this criterion was used only a minority of patients with hypercholesterolaemia would have to be checked. A similar argument could be made for tests for other causes of secondary hyperlipidaemia.

The patient does not seem to have had his liver emzyme and creatinine kinase activities checked before treatment with simavastatin was started. For a patient who is likely to require treatment for several years it is prudent to obtain baseline liver enzyme activities (recommended in the British National Formulary) and creatine kinase activities before treatment is started. The liver enzyme activities would help to identify secondary causes of hyperlipidaemia related to the liver, and raised creatine kinase activity would be identified; the clinician would then be alerted to existing abnormalities that might be influenced by lipid lowering drugs.

Finally, it might have been better to use the term biochemical hyperthyroidism rather than myxoedema to describe the thyroid status of the patient. After all, the patient did not have signs or symptoms to support the diagnosis.

Friarage Hospital,

Northallerton

North Yorkshire DL6 1JG

1 Al-Jubouri MA, Briston PG, Sinclair D, Chinn RH, Young RM Myxoedema revealed by simvastatin induced myopathy. $B M \mathcal{F}$ 1994;308:580. (26 February.)

2 Series J, Biggart EM, O'Reilly DS, Packard CJ, Shepherd J. Thyroid dysfuction and hypercholesterolaemia in the general population of Glasgow, Scotland. Clin Chim Acta 1988;172: 217-21.

\section{Human parvovirus B19}

EDrToR,-After investigating an outbreak in December 1993 of 24 cases of infection with parvovirus B19 in primary schools in Dublin, we wish to comment on J R Pattison's editorial.' Firstly, Pattison noted that parvovirus B19 infection is often misdiagnosed as allergy, rubella, or other "viral infection" and may be clinically recognised only in an outbreak. The most striking feature reported in the case we investigated was the evanescence of the rash (which usually but not exclusively affected the face). The rash flared up in minutes and faded over a few hours only to reappear some hours or days later. Perhaps this feature could be a useful clinical marker that might help to distinguish parvovirus B19 infection from other causes of rashes.

Secondly, Pattison advised caution in the use of commercial diagnostic kits. We initially tested sera from seven children for parvovirus B19 IgM with an immunofluorescence test made in house and found them to be weakly positive. The test was made from a recombinant baculovirus expressing VP1 protein of parvovirus B19 at a dilution of 1:10 after adsorption with goat antibodies to human IgG (Gull Sorb). However, a strong parvovirus B19 IgG reaction was found on western blotting for IgG specific to the VP1 and VP2 proteins of parvovirus B19 prepared in house by recombinant baculoviruses. The immunofluorescence test was there- 
fore repeated at a serum dilution of 1:20 after absorbing with double the quantity of anti-G antibodies, and unequivocal positive results for parvovirus B19 IgM were then obtained. It would be unfortunate if high concentrations of parvovirus B19 IgG were to mask the laboratory confirmation of acute infection with less sensitive techniques when the diagnosis was suspected clinically.

With regard to the management of an outbreak of parvovirus B19 infection, we have prepared a fact sheet for professionals and an explanatory leaflet for parents which we are happy to make available.

Eastern Health Board, Community Care, Vergemount Hall,

\section{Dublin 6,}

Republic of Ireland

Regional Virus Laboratory,

Royal Victoria Hospital,

Belfast BT12 6BN

1 Pattison JR. Human parvovirus B19. BMF 1994;308:149-50. (15 January.)

\section{Consider short courses of antibiotics}

EDrToR,-The Department of Health's determination to reduce prescribing costs will leave many general practitioners looking for ways to do this without harming their patients; perhaps they should consider the duration of the courses of antibiotics that they prescribe for uncomplicated infections. The preferred duration may occasionally be three or even 10 days, but generally it is either five or seven days-though there is no reason to believe that seven days is ever better than five. A study that we have conducted indicates the confusion surrounding this issue and the cost of the two extra days' treatment.

We obtained data from the Prescription Pricing Authority for 1992-3 for all prescriptions for tablets or capsules of amoxycillin, ampicillin, cephalexin, and erythromycin at $250 \mathrm{mg}$ and 500 mg strengths (omitting ethinyl succinate erythromycins); phenoxymethylpenicillin $250 \mathrm{mg}$; trimethoprim $200 \mathrm{mg}$; and co-trimoxazole $400 / 80$ mg. We analysed only items prescribed for short durations and categorised them as five day, seven day, or other by applying standard dosagefrequency to the size of the item: 21 and 15 capsules were commonest for amoxycillin $250 \mathrm{mg}$, and we took these as seven and five day courses respectively.

Nationally, $36.8 \%$ of courses were for five days (5149001/13973878) and $47 \cdot 1 \%$ for seven days (6576613/13973878). Only for phenoxymethylpenicillin did five day courses clearly exceed seven day courses, though for ampicillin the split was roughly even. There was great variation among regions (with five day courses predominating in South Western and Wessex) and among family health services authorities.

To look at variation among doctors we studied singlehanded practices and excluded items known to have been given by deputies, locums, and trainees. For 1817 practices there were 816062 items, with seven day courses again more common except in the case of phenoxymethylpenicillin. Analysis of the 11 preparations combined showed that most practices used both durations of courses, but for the individual preparations there was considerable polarisation. Doctors varied the duration according to which antibiotic they gave but differed over which antibiotic should have which duration. Doctors in partnerships probably behave like their singlehanded colleagues in this respect.

Nationally the cost of the extra two days'

treatment lay between $£ 1.9 \mathrm{~m}$ and $£ 7.2 \mathrm{~m}$, depending on whether it was calculated on the basis of generic or brand leader prices. Had we included the liquid formulations and every other antibiotic, the cost would have been much higher. Some antibiotics are packaged as seven day courses, and this encourages doctors to waste money; original pack dispensing is likely to result in even more waste.

CONRAD M HARRIS Director

Prescribing Research Unit, University of Leeds, Leeds LS2 9NZ

\section{Adult epiglottitis}

\section{Cefuroxime is effective}

EDITOR,-Jonathan H Raphael comments on the likely aetiology and antibiotic treatment of epiglottitis in adults. ${ }^{1} \mathrm{He}$ describes a case caused by Streptococcus pyogenes in which improvement occurred only after benzylpenicillin was added to cefuroxime. The organism "proved sensitive to benzylpenicillin but resistant to cefuroxime." While no one would question that benzylpenicillin is the antibiotic of choice for serious infections due to $S$ pyogenes, the author does not make clear whether he is describing clinical or microbiological failure with cefuroxime; the two are very different. He does not mention antibiotic doses to permit comment on clinical failure. Nor does he state how it was decided that the organism was resistant to cefuroxime. $S$ pyogenes is exquisitely sensitive in vitro to both benzylpenicillin and cefuroxime, with minimum inhibitory concentrations of the order of 0.007 and $0.06 \mathrm{mg} / \mathrm{l}$ respectively. The recommended breakpoint minimum inhibitory concentration for cefuroxime is $4 \mathrm{mg} / \mathrm{l}^{2}$ If the minimum inhibitory concentration of this isolate is considerably higher than the figures quoted here then it is of great interest to microbiologists and clinicians.

Empirical treatment of any serious infection must cover the most likely pathogens. Cefuroxime would provide that cover in adult epiglottitis. Once an organism has been isolated, treatment may need to be changed to the most appropriate antibiotic for that organism. Clinical failures can occur with any antibiotic but must be distinguished from in vitro resistance.

KEITH F BARKER Consultant microbiologist BHARAT PATEL Senior registrar, microbiolog Public Health Laboratory and Department of Microbiology, Central Middlesex Hospital NHS Trust, London NW10 7NS

1 Raphael JH. Adult epiglottitis. $B M{ }^{\gamma}$ 1994;308:719. (12 March.) 2 British Society for Antimicrobial Chemotherapy. A guide to sensitivity testing. I Antimicrob Chemother 1991;27(suppl D).

\section{Seek microbiological advice}

EDITOR,-I was both surprised and dismayed to read the brief case report presented by Jonathan $\mathbf{H}$ Raphael. ${ }^{1}$ My surprise related to his erroneous microbiological statement and my dismay to the lack of consultation with the local microbiology laboratory before publication of his letter.

Any microbiologist would recognise that $\beta$ haemolytic streptococci group $A$ are sensitive to both cefuroxime and benzylpenicillin and would not report them as resistant to cefuroxime. The argument that antibiotics effective against Haemophilus influenzae are insufficient in adult epiglottitis is therefore incorrect and not supported by this case. Serious sepsis, such as epiglottitis in an adult, should ideally be managed in consultation with a microbiologist. Any publication including microbiological data and their interpretation should also involve the microbiologist.

ANDREW SWANN Consultant microbiologist

Joint NHS/PHLS Microbiology Laboratory, Leicester Royal Infirmary, Leicester Royal Infirm

1 Raphael JH. Adult epiglottitis. BMF 1994;308:719. (12 March.)

\section{Reye's syndrome}

EDrToR,-We agree with John F T Glasgow and Raymond Moore that Reye's syndrome is a heterogeneous disorder. ${ }^{1}$ Indeed, it is a non-specific clinicopathological entity, the microvesicular lipid accumulations evident in the liver on light microscopy now being considered to be a non-specific finding for which there are many other causes. ${ }^{12}$ Electron microscopy is now recommended, but in the epidemiological studies reported it has been done in relatively few cases. With regard to aetiology, it is clear that the diagnosis of Reye's syndrome has been revised recently in favour of an inherited metabolic disorder in many patients; other patients are nowadays more correctly diagnosed as having viral disease or an escalation of symptoms induced by antiemetics, whose side effects are now better recognised. ${ }^{2}$

This improved diagnosis, however, implies that the American and British epidemiological studies that suggested a link between Reye's syndrome and aspirin were done on a heterogeneous group of children with different diseases. This fact alone weakens their hypothesis. In the studies carried out by the Centers for Disease Control only the drugs used before the onset of severe vomiting were registered; thus drugs-for example, antiemetics-given between the onset of vomiting and admission to hospital were excluded. Moreover, the Ohio survey first started by registering al drugs taken throughout the illness, but when these data indicated that not only the use of aspirin but also that of phenothiazines and trimethobenzamide hydrochloride was significantly greater in cases of Reye's syndrome than controls the questionnaire was revised. ${ }^{3}$ Only the drugs given before the onset of vomiting were then registered, and thus the same bias was introduced as in the other surveys.

Defining the day of onset of severe vomiting as the onset of Reye's syndrome is arbitrary and results in incorrect data on use of drugs. This arbitrary definition is the key to the whole theory. Indeed, there is no proof that vomiting reflects the early stages of cerebral oedema or the onset of Reye's syndrome; vomiting is often a symptom inherent to the viral infection. ${ }^{2}$ And in some patients the "encephalopathy" was misleading extrapyramidal reactions induced by antiemetics. ${ }^{2}$

In the British risk factor study the use of antiemetics was significantly higher in patients with Reye's syndrome than in the comparison group. 4 Indeed, of 106 patients with Reye's syndrome, 33 had taken at least one antiemetic or antihistamine, or both, before admission compared with 17 of the 185 control patients ( $P<0.0001)$; of these, 15 patients with Reye's syndrome versus three controls had taken an antiemetic such as metoclopramide $(P=0.0001)$. $^{\text {s }}$ (S Hall, personal communication). The British data and the analysis of the Ohio study ${ }^{3}$ show that not only the use of aspirin but also the use of antiemetics, phenothiazines, or other antihistamines is significantly greater in cases than controls.

Why then do people repeat that the only link is the one between aspirin and Reye's syndrome? Would it not be more logical to point to the antiemetics known to be neurotoxic? Their possible role was suggested by the Food and Drug Administration in 1976. ${ }^{5}$ The apparent decline of Reye's syndrome is not a factor in favour of a link with aspirin as increased recognition of metabolic, 